\title{
USER INTERFACE FOR OPTICAL MULTI-SENSORIAL MEASUREMENTS AT EXTRUDED PROFILES
}

\author{
Albert Weckenmann ${ }^{1}$, Johannes Bernstein $^{1}$ \\ ${ }^{1}$ Chair Quality Management and Manufacturing Metrology, University Erlangen-Nuremberg, 91052 Erlangen, \\ Germany, bernstein@qfm.uni-erlangen.de
}

\begin{abstract}
Nowadays the process-control of concave extruding is a measuring task with rising requirements. A novel optical bi-sensorial measurement system - consisting of a shadow- and a light-section-system - as well as suitable methods of analysis for the in-line inspection are presented. The proposals help to ensure the product quality on a higher level than before. The combination of dimensional accuracy and data-density leads to excellent results. The optical multisensor measurement system has to be calibrated and aligned to detect the same surface zone despite of high refresh rates and optical resolutions. The metered characteristics will be coordinate transformed to extrinsic world-coordinates for evaluating form deviations of complex parts. An appropriate user-interface enables to re-calculate measurement objects in-line and evaluate the conformity of the part consequently. Finally the real length information assists to influence the process control. After successful test in laboratory the results will be proved in production to the target: measurement uncertainty of better $0.1 \mathrm{~mm}$ at every profile.
\end{abstract}

Keywords: Laser Scanning, Optical Multi-SensorMetrology, Datafusion

\section{INTRODUCTION}

The optical non-contact manufacturing metrology with high speed qualifies for different materials and ambient environments. Unfortunately, the present optical sensor systems cannot deal with all performances the customer of the parts demands for. On the one hand shadow systems are very accurate but unable to measure concave profiles. They serve only with two-point information of the objects' outline with one diameter. They reach rapid refresh rates of more than $500 \mathrm{~Hz}$ and resolutions of $0.01 \mathrm{~mm} \mathrm{[1].} \mathrm{On} \mathrm{the} \mathrm{other}$ hand the light-section systems can measure concave profiles and complex zones in basic, but not with required accuracies of better than $0.1 \mathrm{~mm}$. Their refresh rate exceed rarely some $\mathrm{Hz}$ what can be too short for in-line applications. An optical multi-sensor measurement system - combining either leads to better measurement results, see Fig. 1.

\section{REQUIREMENTS OF THE SYSTEM}

In order to accomplish the requirement of high accuracy it is to operate with suitable sensors, a flexible and stiff construction and an efficient solution for the data analysis. The parameters of the sensors must bear ambient conditions like temperature, vibration, dust, humidity and infrared radiation [2].

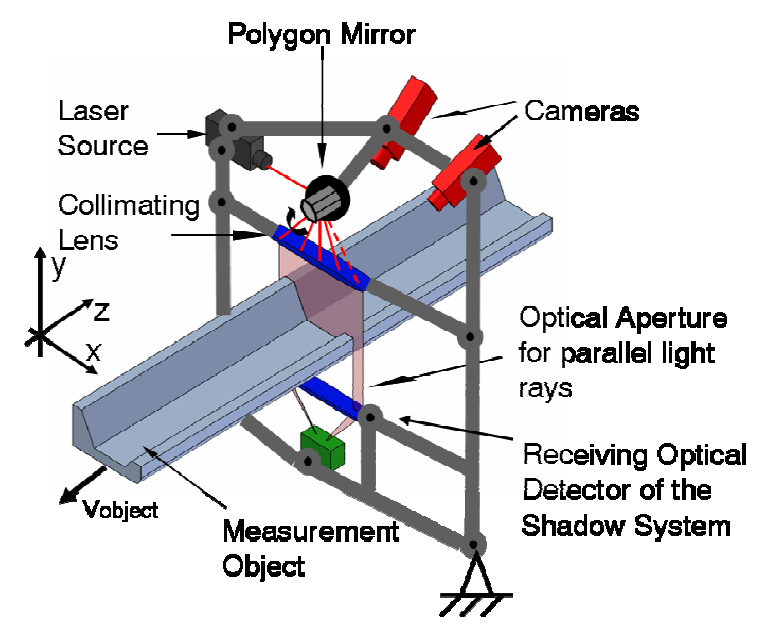

Fig. 1. Conception of the measurement system.

\subsection{Illumination}

As illumination source a semiconductor laser is best suited because of the optical stability, fair price and at the same time less needed space and warm-up time. Blue and similarly green light (wavelengths between $420 \mathrm{~nm}$ to $530 \mathrm{~nm}$ ) are pricy and dangerous for human eyes of participant operators. The red laser (about $660 \mathrm{~nm}$ wavelength) is affordable, less dangerous and available with high power. But the vicinity on spectrum near to the infrared light could be a problem due to the loss of contrast at the image what leads to inaccuracy and accordingly to bad measurements [3]. A performed worst case simulation brought out, that red light is adequate for this application. Finally, $50 \mathrm{~mW}$ light power and an angle of the light fan of $40^{\circ}$ ensure a sharp and strong line projection despite of heat radiation. The displacement to the sensor is important for cooling practises in the shop floor later on and the width wide enough for the device under test, see under 2.4. Another topic is the challenging use of three lasers and to get them in on plane for optimal collaboration and less uncertainties (see 5.6). 


\subsection{Observance of the object}

The projected laser line must be observed stationary and has to reach accuracies down to $0.1 \mathrm{~mm}$, but a camera with sensitivity, high resolution and small need of space is suited. The refresh rate must be over 20 frames per second (fps) to ensure at least one grab every $250 \mathrm{~mm}$ (the profiles extrudes with velocities up to $8 \mathrm{~m} / \mathrm{s}$ ). With respect to great light intensity a CCD-Chip with 1920 to 1080 pixels was selected. The wide band format is perfect to measure medium distorted lines on complex profile shapes. Filters against UV and infrared radiation help to separate the disturbances. The cameras are freely adjustable in angle and distance.

\subsection{Measurement objects}

The objects are several extruded profiles from brass, aluminium and polymers. They are endless semi-finished products from $10 \mathrm{~mm}$ to $100 \mathrm{~mm}$ diameter and used in a couple of technical applications. As a result the end customer does not have to rework in the same amount to usual raw material. The profiles have temperatures of 800 ${ }^{\circ} \mathrm{C}$ and move non-constant in speed. Unknown frequencies and shocks from the process complicate the measuring place. Lubricant, dust and oxide particles are constraints for the optical system in addition. At all, there are different forms to measure: H-, L-, T-, round-, hexagonal-profiles and some special types.

\subsection{Reconstruction of the part contour}

The grabbed images have to be recorded concerning to the form deviation of the object. Normal triangulation works only with two angles between the object and the observer for easy reconstruction, see Fig. 2.

$\Delta h=\Delta l \frac{1}{\sin \beta \cdot(\cot \alpha+\cot \beta)}$

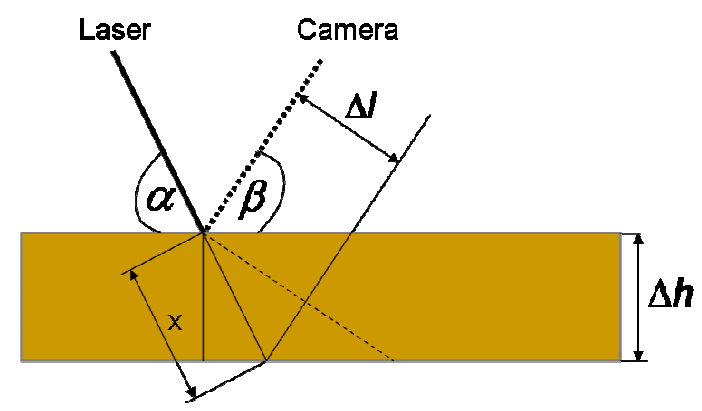

Fig. 2. Fundamental triangulation with one light-section system

On the one hand the light section systems have to be adjusted with respect to the different profiles. On the other hand only known and steady relations of distance and angle can lead to accurate reconstruction by triangulation. At all, the reconstruction does not allow to effort much time because of real time abilities. For instance by serving with $20 \mathrm{fps}$, one measurement with all operations has only $50 \mathrm{~ms}$ time to be proceeded obviously. For this reasons an extensive calibration concept had to be formulated and implemented, see 4.3 [4].

\section{DESIGN OF A TEST STAND}

The system consists of two sensor systems which have to combine the advantages of both methods optimally. For testing and evaluating the reachable performance a test stand has been constructed, see Fig. 3. The frame is made of aluminum, the light section systems - cameras and lasers are fully adjustable around the part.

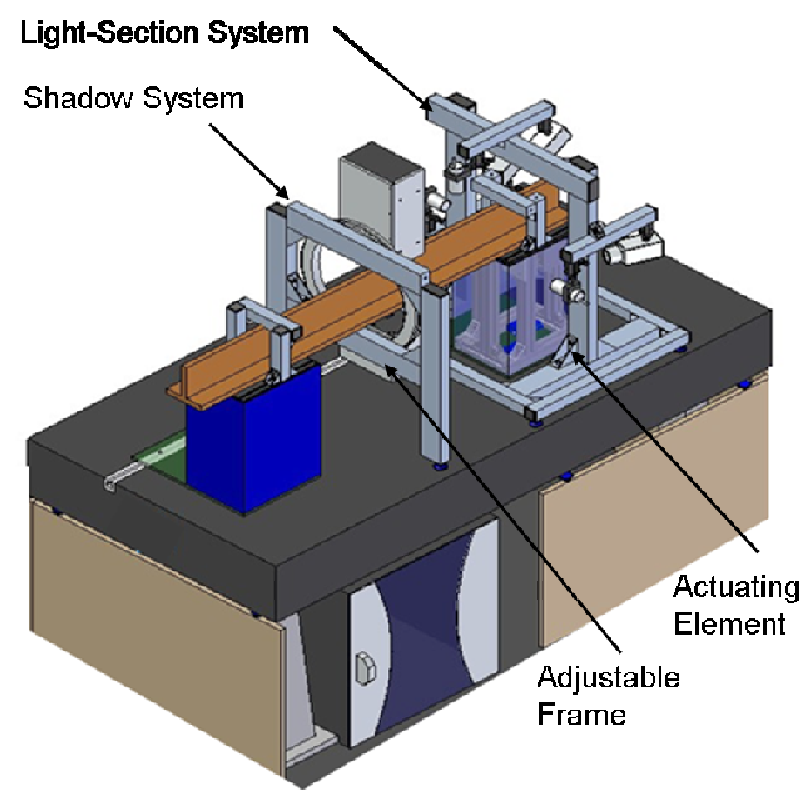

Fig. 3. Mock-up of the optical bi-sensorial test stand

\subsection{Arrangement}

There are three sub-systems built in to ensure a $360^{\circ}$ view around complex shaped or concave profiles. For this reasons an adjustment concept for every profile size and type helps to install, adjust and calibrate the test stand for different sizes and types again. The distances of the several lasers, their angle, the distance of all cameras, their angle and the distance between the cameras and the lasers have to be respected [5]. To follow the parameters operatively and accurately a calibration procedure with various steps must be fulfilled, see 4.3 .

\subsection{Main Elements}

Flexible knob holders allow to adjust and to fix all sensors in two rotatory and two translational degrees of freedom. The measurand can be changed easily and hold or moved automatically in two dimensions isochronous by actuating elements. Linear motors with stored program control (SPC) serve with power for profile pieces up to $60 \mathrm{~kg}$ and frequencies from $0.1 \mathrm{~Hz}$ to $5 \mathrm{~Hz}$. This movement simulates the real vibrations and shocks in the shop floor. The shadow system is easily and fully rotatable $-360^{\circ}$ around the part. The shadow system can be adjusted depending on different profile types for ensuring the best view, too. A workstation with customized software conjoining flexible libraries of high-level language, ensure the image acquisition, the calibration, all calculations and the merging. 


\section{MEASUREMENTS}

All systems' sensors have to be motivated, images have to be read out and datasets have to be stored in a history. Afterwards the calibration, merging and reconstruction for the evaluation of form deviation are to proceed.

\subsection{Light-section subsystem}

For a full view more than one sensor pair is needed (see 3.). Three ones are an agreement between costs, effort and the possibility of measuring complex zones. The Images from every camera are triggered by a master signal, see Fig. 4a. Every picture can be sized and enhanced by contrast, Fig. 4b. The Edge Finder localizes the contrast of the laser and saves it in arrays, Fig. 4c. The three sensor pairs are installed with an optical overlap of about $10 \%$ to ensure the reconstruction to one form by an algorithms perfectly. The calibration of every profile is done by protractor for the lasers and by gauge blocks and by calibration plates for the cameras distortions and distances.

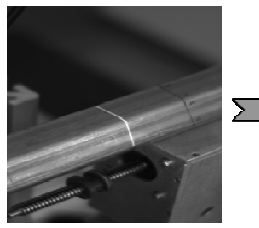

(a)

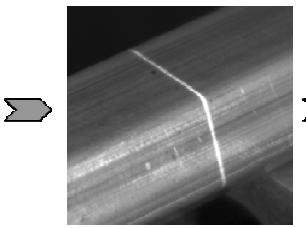

(b)

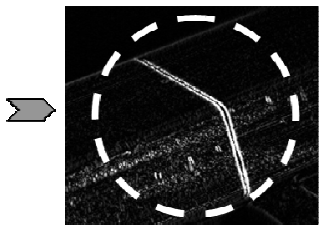

(c)
Fig. 4. Grabbed film by the light-section system under movement

\subsection{Shadow subsystem}

The shadow system deals with scalar length information of the maximum outline of the profile by reducing the task to timing. The system has to be calibrated by two-point information. The linear calibration is done by two different sizes of gauge blocks and compensates the offset error and the pitch error. The binary information is read out directly and can be used for the full calibration and the outlines' speed of the data stream.

\subsection{Calibration of the combined system}

The calibration of all sensors demands for global extrinsic coordinate systems. It has to be built together, calibrated for every type in the catalogue and aligned after to one scatterplot for serving with authentic proposals of measurement uncertainty. The integration with a fluctuating point of origin has to be provided. The calibration with many degrees of freedom for the conjoined optical measurement system can be done by theodolites, gauge blocks and other special artefacts to the traceability of the unit of length, the meter.

\subsection{Datafusion by combining the results}

To combine the three datasets which are calibrated and reconstructed together the ICP-algorithm, (Iterative closest point) which is available in different variants can be used. The overlapped zones are used to straighten and align the datasets. After that the more accurate length of the outline by the shadow system can be weighted and calculated with the result of the merged light-section point-cloud at zones near to the maximum width. Finally, the results can be analyzed and evaluated.

\section{USER INTERFACE}

The operator must easily use and analyse the reconstruction and the measurement results. He must be guided through the measurement and help has to be provided if needed to ensure stability for the process control in-line.

\subsection{Procedure of reconstructing shaped elements}

All extruding profiles must be compared with a reference which normally comes from the technical drawing. The reconstruction of the measured point-cloud with a comfortable graph and the visualization of the measured contour as well as comparing them to a reference - in due to evaluate the conformity or non-conformity of the part needs an intuitive user-interface implicitly, Fig. 5. It can be selected to which criteria shall be reconstructed. Implemented for the circles and loop structures are Gaussian, Inscribed, Maximum, Minimum method and, very important for many applications - the Circumscribed Maximum [6] [7]. Other methods provided are e.g. radius and line structures. For all visualization, calculations and experiments the ability of calculation time and accuracy must be ensured, see 5.4 and 5.5.
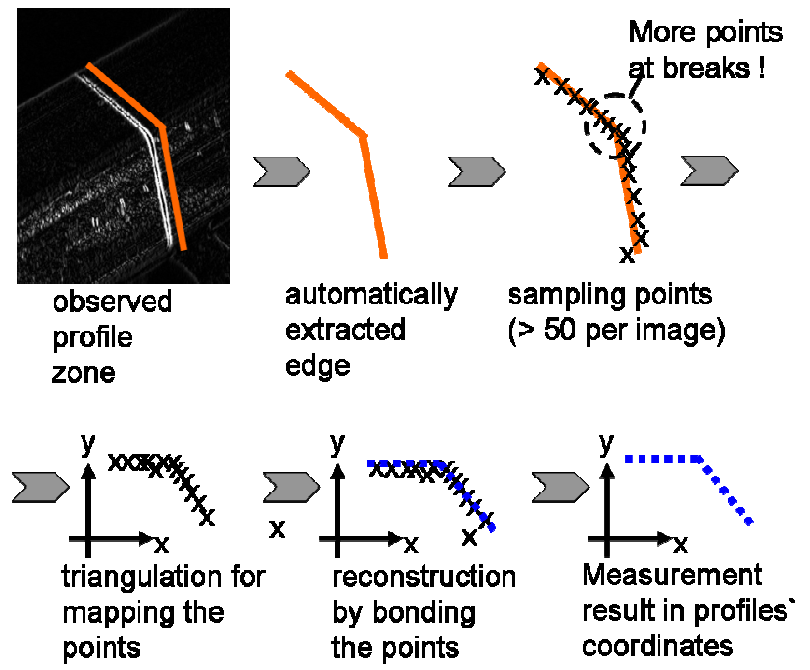

Fig. 5. Image processing and reconstruction in arrays

\subsection{Evaluation of the measurement results}

The user-interface shows the reference dataset of any profile and the measured one with all calculated sampling points. Different colours help to divide into "conformance" or "non conformance" sections and help to control the process performance indicators, the machine capability and the process quality. Form deviations, tolerances from length, form and position after DIN ISO 1101 can be analyzed and evaluated [8] in real-time during the process. The connection to the process control is enabled. 


\subsection{Guidance on operator}

A customized help function, which blocks unknown windows and provides safety against "miss-use", ignoring wrong inputs by keyboard and an ergonomic user-interface design conform to DIN ISO 9241-10 und -17 ensures optimal usability, Fig. 6.

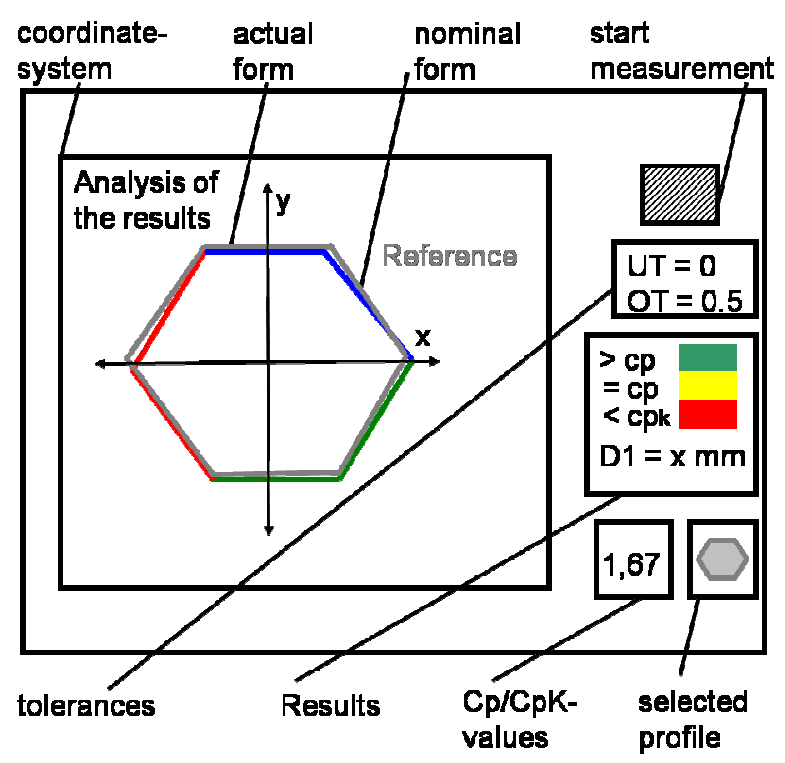

Fig. 6. User Interface for the multi-sensor measurement system

\subsection{Installation concept of different profile types}

To ensure that a variety of profiles can be measured it is necessary to adjust every sensor and calibrate them after changing the type or size of the measurement object. To optimize the adjustment and not to lose much time a concept was implemented which helps to install every sensor from best angle and distance. It is also possible to give out areas which can probably not be illuminated or observed optimally. For this reasons the physically given parameters of the lasers and cameras have to be calculated with respect to the measurement object in different variations. The target: full view $360^{\circ}$ around using the lasers L1, L2, and L3 in obtuse angles to the objects and not to fall below a distance of $200 \mathrm{~mm}$ to the object because of the depth of field of the illumination and cameras, Fig.7.

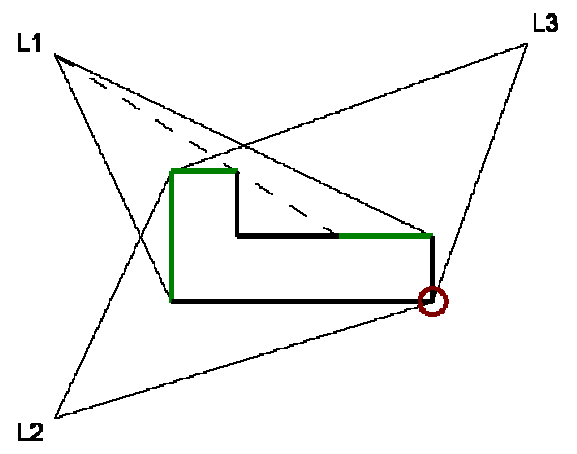

Fig. 7 Illumination by three lasers of a L-profile, red: Shaded or critical area, black: illuminated area by one laser, green: illuminated area by more than one laser
At complex profiles like $\mathrm{T}$ - or $\mathrm{H}$ - there can be areas which are not recordable because of no or not sufficient overlaps (of about at least $10 \%$ ) for the data fusion after. Thus areas have to be avoided and, if not possible, the operator has to be warned by the user interface. In Fig. 7 is illustrated the problem which is similarly present for the three cameras as well. In case of an L-profile it is always possible to illuminate and observes the whole profile, but one corner is not overlapped and must be the fitting section for the fusion algorithm.

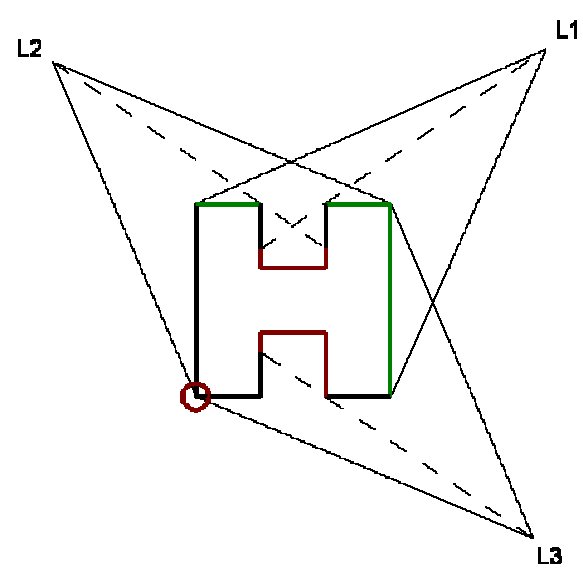

Fig. 8 Illumination by three lasers of a H-profile, red: Shaded Areas, black: illuminated area by one laser, green: illuminated area by more than one laser

At an H-profile in Fig. 8 it is not possible with three systems to illuminate and observe the whole profile. Indeed one corner is not visible and must be the fitting section for the fusion algorithm. The inner sections can not be recorded in that configuration. For this use, the system hast to be readjusted again but the H-profile cannot be measured $360^{\circ}$ with one configuration at every section. As a result it has to be chosen which section is most important. For regions of most interest (e.g. in the inner areas of the profile) the system should be re-adjusted to smaller angles between L1 and L2 what leads on the other hand to the disadvantage: border contours cannot be measured at every section.

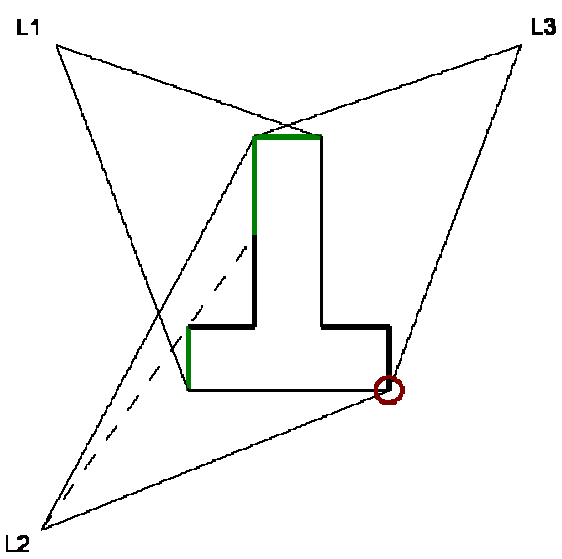

Fig. 9 Illumination by three lasers of a T-profile, red: Shaded area, black: illuminated area by one laser, green: illuminated area by more than one laser 
The T-profile can be measured in normal ratio (of shaft and upper part) up to $100 \mathrm{~mm}$ diameter. Only one point the data sets will be fitted together - is critical and can lead to a non-defined situation, Fig. 9.

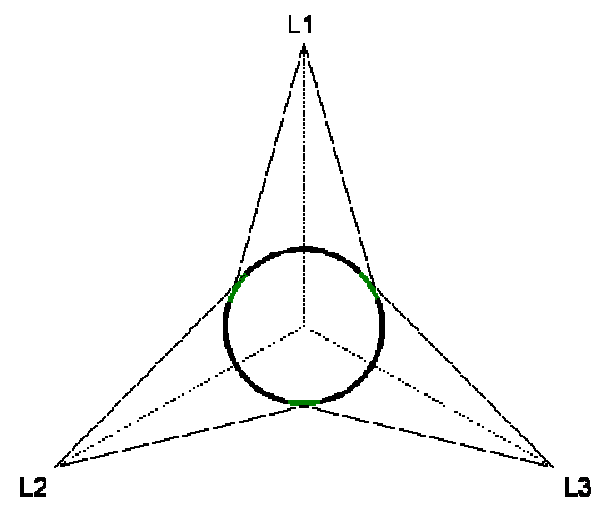

Fig.10 Illumination by three lasers of a round profile, black: illuminated area by one laser, green: illuminated area by more than one laser

The round profile can be measured without shaded zones up to $100 \mathrm{~mm}$ diameter. There are no critical sections. Normally a rotational distance of $120^{\circ}$ between the three lasers is to be used, Fig. 10.

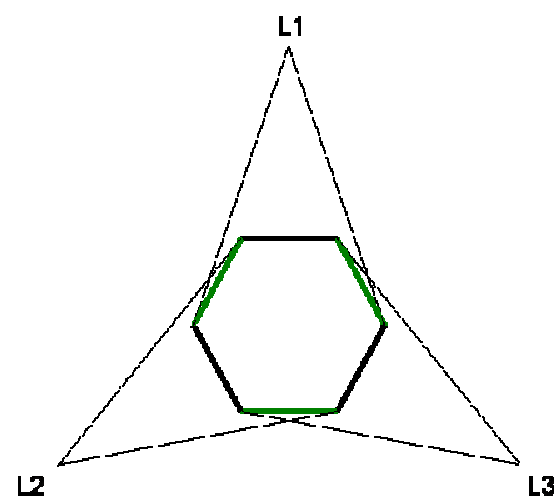

Fig.11 Illumination by three lasers of a hexagonal profile, black: illuminated area by one laser, green: illuminated area by more than one laser

The hexagonal profile can be measured up to $100 \mathrm{~mm}$ diameter without problematic zones. There are no theoretically critic sections but the light rays run nearly parallel in some areas which lead to low accuracy in reconstruction, Fig.11. Normally an angle of $120^{\circ}$ between the lasers is to be applied. In further research more systems could improve this circumstance for complex profiles but that is expensive and the collision of mechanical installation by more systems increases other disadvantages like complexity of the data fusion and loss of calculation speed. The square profile can be measured at every section close to the T-profile or the L-profile (there is one suboptimal measurable point only), Fig. 12. The area of overlap regards to accurate installation because the two lines have to match without loss of sharpness and contrast of the laser line.

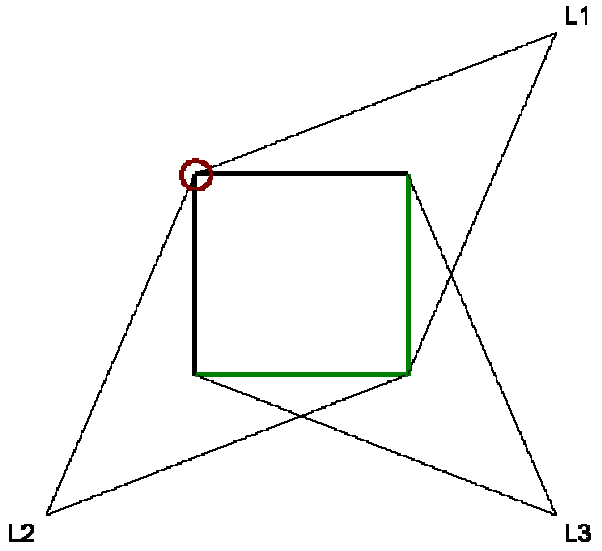

Fig.12 Illumination of a square profile by three lasers, red: Shaded areas, black: illuminated area by one laser, green: illuminated area by more than one laser

At all the user-interface gives automatically the recommendation how the sensors should be installed.

\subsection{Calculation time}

The calculation time depends on the profile and the density of points per reconstruction [9]. Every measuring point has to be positioned at the reconstruction by comparing to all others and collected the distance to the normal vector of the chosen form element. E.g. the circumscribed maximum circle with " $n$ " points has as many possibilities "P":

$$
\begin{aligned}
& P=(n-3) \cdot \frac{n !}{3 !(n-3) !}=(n-3) \cdot\left(\frac{n}{3}\right) \\
& \stackrel{(2)}{\longrightarrow} \text { for }(n=50), P=\left(47 \cdot \frac{50}{3}\right) \approx 783
\end{aligned}
$$

Neglecting the most complex profile types, like "T" or "H", $30 \mathrm{~ms}$ calculating time are enough to process 100 points per dataset. The hardware based time for image grabbing and the interface deceleration are smaller than $10 \mathrm{~ms}$ time. In sum of $40 \mathrm{~ms}$ (at all) finally the measurement allows $20 \mathrm{fps}$. So, the cameras can be fully utilized.

\subsection{Accuracy of the calculation}

The data fusion is - more than one magnitude - better than it is necessary for the agglomerated accuracy of the multi-sensorial optical measurement system, Fig. 13.
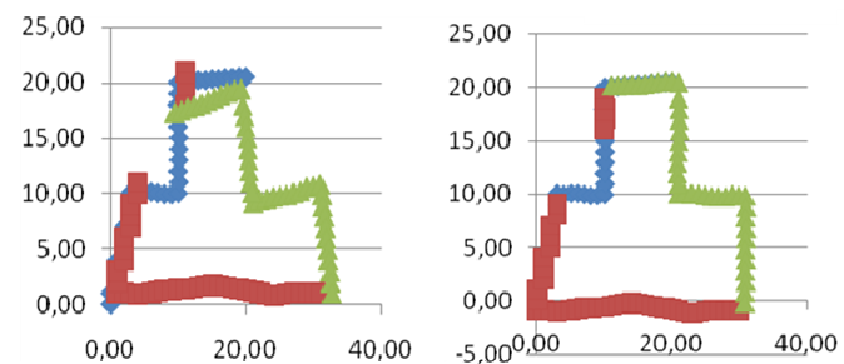

Fig. 13 Data Fusion in principle before (left) and after (right) using gaussian algorithm, here for a T-profile in $\mathrm{mm}$ 
The complexity of the different calculations varies, accordingly the number of achievable calculations per section is between $5 \times 10^{3}$ up to $8 \times 10^{4}$. The resulting deviation in maximum - at off-line tests by simulation - had been smaller than $0.01 \mathrm{~mm}$ and can be improved by more subset points for the reconstruction and analysis anytime. Another topic for declaring the accuracy of the user interface and the algorithms is how many points of the grabbed and reconstructed points are effectively used for the reconstruction of characteristics (length and form).

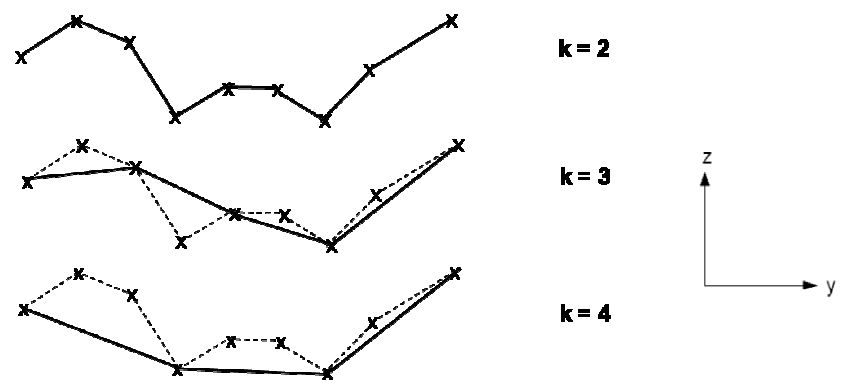

Fig. 14 Principle loss of accuracy by using less points for more speed in measurement

By systematically neglecting points for linear increasing calculation speed, the accuracy of the contour and finally the measurement results of the characteristics (length, form, parallelism) decreases, see Fig. 14.

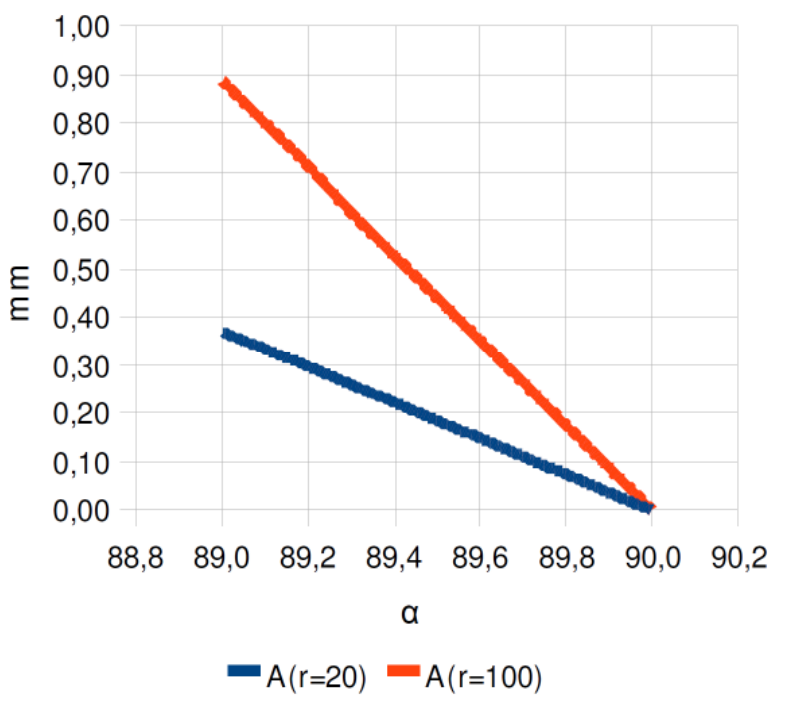

Fig. 15 Resulting deviation (in $\mathrm{mm}$ ) by cosines error of the angle (in degree) between camera and laser of the light section systems, blue: $20 \mathrm{~mm}$ objects' diameter, red: $100 \mathrm{~mm}$ objects' diameter

Typically, the angle cannot be adjusted to $90^{\circ}$ perfectly. As a result a systematic form deviation of the projected line is given and finally an inaccurate reconstruction of the part. To minimize it at the best three micrometer bolts help to bring the three lasers in one plane with inaccuracies less than $0.2^{\circ}$ and as a result effects of less $0.08 \mathrm{~mm}$ what can be tolerated at most. They are adjustable on $0.01 \mathrm{~mm}$ steps in rotational and translational degrees of freedom, Fig. 15.

\section{CONCLUSION AND OUTLOOK}

The introduced multi-sensorial measurement system consists of an optical light-section system and a shading system which should be merged to combined information. The objective to align high data rates with accuracy and the possibility to measure concave profiles is partly achieved. All sensor systems are running on a workstation with adapted software. A custom designed user-interface helps to receive robust, quantitative, reconstructed measurement results of a $360^{\circ}$ view at complex profiles and analyzes them optimally in-line, despite of rough constraints. The referenced evaluation of length and form is directly and ergonomically usable to select in conformity and nonconformity in real-time, 20 times a second. The next steps are to fine the sensor-calibration, propose the measurement uncertainty numerically, to optimize the results and the measurement process plus improving the juncture between hardware and software. After that, the optical multisensorial measurement system will be installed to the shop floor where robustness and many influences will be a critic. It assists to monitor the process in real-time better than before what leads to superior product quality and less rework at the end customer of the semi-finished products.

\section{ACKNOWLEDGMENTS}

This research is gratefully founded by the Bavarian Research Foundation (BFS) in the project "Optical BiSensor-Measurement System for the Production Control of Extruded Profiles".

\section{REFERENCES}

[1] Bauer, N., "Compendium to image-guided sensor technology“ (,Leitfaden zur bildgebenden Sensortechnik“), Fraunhofer, Erlangen, 2003.

[2] Trostmann, E., "Compendium to Fundamentals and Applications of optical 3D-Metrology“ (,Leitfaden zu Grundlagen und Anwendungen der optischen 3-D Messtechnik“), Erlangen, Fraunhofer, 2003

[3] Pfeifer, N., Briese, C., "Laser Scanning - Principles and Applications", Moskau, 2007

[4] Wagner, T., "Automated Configuration of Image Processing Units“ (,Automatische Konfiguration von Bildverarbeitungssystemen“), Erlangen-Nuremberg, 2000

[5] Hoffmann, J., ,User Guide of Metrology“ („Handbuch der Messtechnik“), Munich, Hanser, 2001

[6] Weckenmann, A., Eitzert, H., Weber, H., „Coordinate Metrology evaluating function-oriented“ ("Koordinatenmesstechnik funktionsorientiert auswerten"), Conference on Microtechnology, VDI, 1992

[7] Goch, G, Lübke, K, "Tschebyscheff approximation for the calculation of maximum inscribed/minimum circumscribed geometry elements and form deviations“, Bremen, BIMAQ, 2008

[8] Weckenmann, A., Eitzert, H., Garmer, M., Weber, H., "Functionality-oriented evaluation and sampling strategy in coordinate metrology“, Hamburg, University of Federal Armed Forces, 1995

[9] Maresch, T., „Mathematics, Conjunction of 2D- and 3Dpointclouds“ (,Mathematik- Verknüpfung von 2D- und 3DPunktwolken”), Giessen, Dissertation, 2006 